DOI: 10.12957/demetra.2018.29094

\title{
Avaliação do estado nutricional de pacientes hospitalizados: uso de métodos convencionais e não convencionais
}

\section{Evaluation of the nutrition status of hospitalized patients: use of conventional and non-conventional methods}

\author{
Juliany Caroline Silva de Sousa' \\ Cristiane Maria de Oliveira? \\ Ana Shirley Marinho Araújo \\ Raíssa Dantas dos Santos \\ Daliana Caldas Pessoa da Silva² \\ Lidiane de Lima Fernandes' \\ Alexandre Coelho Serquiz ${ }^{3}$ \\ ' Laureate International Universities, \\ Universidade Potiguar, Curso de Nutrição, Escola \\ da Saúde. Natal, RN, Brasil. \\ 2 Universidade Federal do Rio Grande do Norte, \\ Programa de Pós-graduação em Ciências da \\ Saúde. Natal, RN, Brasil. \\ ${ }^{3}$ Centro Universitário do Rio Grande do Norte, \\ Curso de Nutrição. Natal, RN, Brasil. \\ Correspondência / Correspondence \\ Alexandre Coelho Serquiz \\ E-mail: alexandreserquiz@gmail.com
}

\section{Resumo}

A avaliação do estado nutricional é essencial no acompanhamento de pacientes internados, pois tem por objetivo verificar as alterações nutricionais, possibilitando uma intervenção adequada para a recuperação e/ou manutenção da saúde dos indivíduos. Este estudo tem por objetivo avaliar o estado nutricional dos pacientes hospitalizados, utilizando os métodos convencionais e não convencionais: espessura do músculo adutor do polegar (EMAP), força da preensão manual (FPM), índice de massa corporal (IMC), perímetro da panturrilha (PP) e perímetro do braço (PB). Trata-se de um estudo transversal, em que foram avaliados 88 pacientes de ambos os sexos. Os resultados apontam que modificação da composição corpórea, a inatividade física, aumento do estado inflamatório, edemas e desnutrição podem estar muitas vezes relacionados com as diferenças dos estados nutricionais verificados a partir dos distintos parâmetros observados, sendo a eutrofia mais prevalente em todos os métodos, exceto nos métodos da EMAP e da FPM principalmente para os idosos, onde predominou o risco de depleção. Sugerese que tanto a MAP quanto a FPM podem ser indicadores que detectam complicações atribuídas a riscos de depleções tanto para a população adulta quanto para a população idosa.

Palavras-chave: Avaliação Nutricional. Antropometria. Desnutrição. 


\section{Abstract}

The assessment of nutritional status is essential in the follow-up of hospitalized patients, since it aims to check fornutritional changes, allowing an adequate intervention for recovery and / or maintenance of individuals' health. This study is aimed at evaluating the nutritional status of hospitalized patients using adductor pollicis muscle thickness (APMT), handgrip strength (HGS), body mass index (BMI), calf circumference (CC) and arm circumference (AC). It is a cross-sectional study, in which 88 patients of both sexes were evaluated. The results indicate that changes in body composition, physical inactivity, increased inflammatory status, edemas and malnutrition may often be related to differences in nutritional status, as verified from the different parameters observed, with normal weightbeing more prevalent in all methods, except in the APMT and HGS methods, mainly for the elderly, where the risk of depletion was predominant. It is suggested that both APMT and HGS may be indicators fordetection ofcomplications attributed to risks of depletions for both the adult and the elderly populations.

Keywords: Nutritional Assessment. Anthropometry. Inpatients.

\section{Introdução}

A avaliação do estado nutricional é essencial no acompanhamento de pacientes internados, pois tem por objetivo verificar as alterações nutricionais, possibilitando uma intervenção adequada para a recuperação e/ou manutenção da saúde dos indivíduos. ${ }^{1}$

Os hospitais devem padronizar técnicas de avaliação do estado nutricional e sistematizar sua aplicação, pois estas detectam o risco de desnutrição hospitalar e auxiliam no tratamento nutricional e/ou melhora do prognóstico do paciente hospitalizado. ${ }^{2}$ Sabendo disso, a antropometria é um importante instrumento de avaliação nutricional, por fornecer os valores de composição corporal, massa magra e tecido adiposo.

Existem diferentes métodos para avaliar a composição corporal, desde as medidas das reservas proteicas e gordurosas por meio da antropometria tradicional ou convencional, até a utilização de equipamentos mais sofisticados. ${ }^{3}$ Os métodos convencionais mais utilizados para avaliação da desnutrição entre adultos e idosos, são: Peso, estatura, índice de massa corporal (IMC), perímetro do braço $(\mathrm{PB})$ e perímetro da panturrilha $(\mathrm{PP}){ }^{4}$ 
Apesar da importância da avaliação muscular na caracterização do estado nutricional, ainda permanecem limitados os métodos para sua aferição direta. ${ }^{5}$ Com isso, há novas técnicas não utilizadas rotineiramente na prática clínica, dentre as quais a espessura do músculo adutor do polegar (EMAP) e a força de preensão manual (FPM). Esta última, utilizada como parâmetro na prática clínica, desempenha papel importante no controle de processos de reabilitação na avaliação e tratamento de desordens músculo-esqueléticas da mão ${ }^{6}$ e na avaliação de pessoas com doenças neurológicas. ${ }^{7}$

Além disso, a FPM é entendida como indicador geral de força e potência musculares ${ }^{8}$, sendo um marcador de massa muscular bastante valorizado para avaliação em pacientes hospitalizados. Uma de suas principais vantagens é o fato de não ser influenciado pelo estado de hidratação do paciente. $^{9}$

Além da FPM, a espessura do músculo adutor do polegar (EMAP) vem sendo aplicada sem limitações à sua execução, imediatamente após a admissão, sendo possível identificar com rapidez, sensibilidade e fidedignidade pacientes desnutridos ou em risco nutricional. Este método possibilita que a instituição elabore um plano nutricional precoce e adequado, e ainda permite o monitoramento adequado do estado nutricional dos pacientes, sendo importante indicador de recuperação do paciente hospitalar. ${ }^{10}$

Os métodos não convencionais apresentados demonstram benefícios quanto à detecção de complicações e riscos advindos de depleções musculares, tornando sua inserção de suma importância na avaliação nutricional. Essa detecção oferece melhor visão do estado nutricional do paciente e, consequentemente, auxilia na articulação de propostas mais rápidas e eficazes no meio hospitalar.

Desta maneira, este estudo tem por objetivo avaliar o estado nutricional de pacientes hospitalizados, utilizando diferentes métodos e técnicas, como: espessura do musculatura adutor do polegar (EMAP), força da preensão manual (FPM), índice de massa corporal (IMC), perímetro da panturrilha $(\mathrm{PP})$ e perímetro do braço $(\mathrm{PB})$.

\section{Metodologia}

Antes de sua realização, o estudo foi submetido ao Comitê de Ética em Pesquisa da Plataforma Brasil e iniciada após sua aprovação, registrada sob o número de protocolo 080376/2013, obedecendo ao cronograma proposto. Os participantes foram informados dos objetivos, riscos e procedimentos envolvidos na pesquisa e aqueles que aceitaram participar assinaram o Termo de Consentimento Livre e Esclarecido (TCLE). 
Trata-se de estudo transversal no qual foram avaliados 88 pacientes, de ambos os sexos, assistidos em hospital público. Dos pacientes selecionados, foi analisado o público adulto e idoso, onde se classificaram como idosos as pessoas acima de 60 anos, segundo a Organização Mundial da Saúde (OMS), e adultos as pessoas entre 19 e 60 anos completos ${ }^{11}$ Os pacientes foram convidados a participar da pesquisa e, após o aceite, foram aplicadas as técnicas descritas na literatura para aferição do $\mathrm{MAP}^{12}, \mathrm{FPM}^{13}, \mathrm{PP}^{14}$ e $\mathrm{PB}^{15}$

Foram incluídos na pesquisa aqueles que se apresentavam com os membros corporais livres no momento da aferição e hospitalizados por pelo menos três dias. Foram excluídos os pacientes recém-admitidos no hospital, apresentando 19 anos incompletos e aqueles incapazes de responder a comandos. Para a avaliação objetiva do estado nutricional, aplicou-se um questionário estruturado para registros dos dados antropométricos no momento da admissão. Após aceitar participar, assinaram o TCLE.

As medidas de peso e estatura foram obtidas através de registro no prontuário do paciente. A partir dos valores de peso e altura, calculou-se o índice de massa corporal (IMC) nos adultos, de acordo com a OMS, ${ }^{16}$ e dos idosos de acordo com Lipschitz. ${ }^{17}$

O estudo teve como variáveis de identificação o número de ordem, e para classificação do estado nutricional, foram considerados os indicadores específicos por faixa etária. O critério utilizado para a classificação de adequação do perímetro da panturrilha foi estar $>31 \mathrm{~cm}^{18}$; para classificação do perímetro do braço, foram usados os critérios de percentil $<5=$ magro, 5 a $15=$ abaixo da média, 16 a 85 = média, 86 a 95 = acima da média, $\geq 95$ = excesso de gordura. ${ }^{19}$ Para classificação da EMAP, utilizando a classificação a partir da mão não dominante, foram utilizados os critérios de $<13,4 \mathrm{~mm}=$ desnutrido e $>13,4 \mathrm{~mm}=$ eutrófico.${ }^{20}$ Para classificação da força da preensão manual (FPM), foram usados os critérios de percentil $\leq 25=$ fraco; $<25$ e $\leq 75=$ mediano; $>75=$ muito bom, sendo considerados em risco de depleção os valores abaixo do percentil 25, e adequados os valores acima de do percentil $25 .{ }^{21}$

Para análise estatística, foi construído um banco de dados utilizando o programa Excel 2010. Após este procedimento, os dados foram exportados para o software SPSS, versão 20.0, para proceder à análise estatística.

Para a análise das variáveis categóricas, foram verificadas as frequências relativas e apresentadas com seus respectivos intervalos de confiança.

As medidas em forma de variáveis contínuas foram testadas quanto à natureza de sua distribuição e apresentadas por meio das medidas de tendência central e de dispersão (média/ mediana e desvio-padrão).

Para a identificação da natureza da distribuição dos dados, foi utilizado o teste de KolmorovSmirnoff, ao nível de significância de $5 \%$. 


\section{Resultados e Discussão}

Os resultados apontam que a população do estudo foi composta por $53,57 \%(n=47)$ de pacientes do sexo masculino e 46,42\% (n=41) do feminino. A média de idade da amostra foi de 53,10 $\pm 21,23$ anos. A média do IMC $\left(\mathrm{Kg} / \mathrm{m}^{2}\right)$ foi de 28,46 \pm 7,04, quando analisada a totalidade da amostra.

A partir dos resultados encontrados através do perímetro da panturrilha $(\mathrm{cm})$, pode-se obter uma média total de 35,38 \pm 4,37, sendo classificada como eutrofia nos diferentes públicos. Reis et al. ${ }^{16}$ também observaram essa maior prevalência na CP, onde 81,2\% dos pacientes encontravamse eutróficos e apenas 11,3\% apresentaram depleção muscular, conforme a região avaliada dos pacientes internados em um hospital geral. Soares \& Mussoi ${ }^{22}$ identificaram que dois em cada cinco idosos tinham a CP inferior a $31 \mathrm{~cm}$. Observa-se que à medida que o estado nutricional é depletado ou preservado, as médias de IMC, perímetro do braço e da panturrilha acompanham o nível do estado nutricional.

Na medida da FPM (mm), constatou-se menor força estática no sexo feminino e o público idoso apresentou maior depleção quando comparado aos adultos. Costa et al., ${ }^{23} \mathrm{em}$ seu estudo com idosos não institucionalizados, mostraram FPM menor no público feminino, sendo que todos apresentaram risco de depleção. A FPM é utilizada para avaliar a força estática de preensão manual do indivíduo, já que a mesma está associada à sua funcionalidade. ${ }^{24}$ Essas mudanças antropométricas geralmente são associadas à perda da força e da resistência muscular, podendo levar a um declínio das capacidades funcionais.

Idade, sexo, etnia, região, tipo de doença associada e estímulo à atividade física são algumas variáveis que interferem na diminuição da massa magra e aumento do percentual de gordura corporal, de modo que essas alterações podem afetar a força estática, por isso a avaliação antropométrica torna-se relevante. ${ }^{25,26}$ As variáveis da avaliação antropométrica, por sexo, estão apresentadas na tabela 1 . 
Tabela 1. Perfil antropométrico dos pacientes internados no hospital de Natal-RN, 2017.

\begin{tabular}{|c|c|c|c|c|c|c|}
\hline \multirow{2}{*}{ Variáveis/Categoria } & \multicolumn{2}{|c|}{ Total } & \multicolumn{2}{|c|}{ Masculino } & \multicolumn{2}{|c|}{ Feminino } \\
\hline & $\mathrm{n}$ & $\%$ & $\mathrm{n}$ & $\%$ & $\mathrm{n}$ & $\%$ \\
\hline Sexo & 88 & 100,0 & 47 & 53,57 & 41 & 46,42 \\
\hline Idade (Média \pm DP) & $53,10 \pm 21,23$ & & $55,88 \pm 21,08$ & & $49,69 \pm 21,7$ & \\
\hline Peso (Média \pm DP) & $77,88 \pm 20,84$ & & $80,83 \pm 22,98$ & & $74,92 \pm 19,00$ & \\
\hline $\operatorname{IMC}\left(\mathrm{Kg} / \mathrm{m}^{2}\right)$ & $28,46 \pm 7,0$ & & $27,75 \pm 6,45$ & & $29,17 \pm 7,80$ & \\
\hline FPM (mm) & $17,96 \pm 16,21$ & & $23,86 \pm 18,6$ & & $9,70 \pm 6,48$ & \\
\hline $\operatorname{EMAP}(\mathrm{mm})$ & $98,75 \pm 40,83$ & & $94,61 \pm 34,80$ & & $103,84 \pm 48,22$ & \\
\hline $\mathrm{PP}(\mathrm{cm})$ & $35,38 \pm 4,37$ & & $35,69 \pm 4,42$ & & $35,00 \pm 4,45$ & \\
\hline
\end{tabular}

Nota: $\mathrm{DP}=$ desvio padrão, $\mathrm{IMC}=$ índice de massa muscular, $\mathrm{FPM}=$ força de preensão manual, $\mathrm{EMAP}=\mathrm{espessura}$ do músculo adutor do polegar; $\mathrm{PP}=$ perímetro da panturrilha

Fonte: Dados da pesquisa.

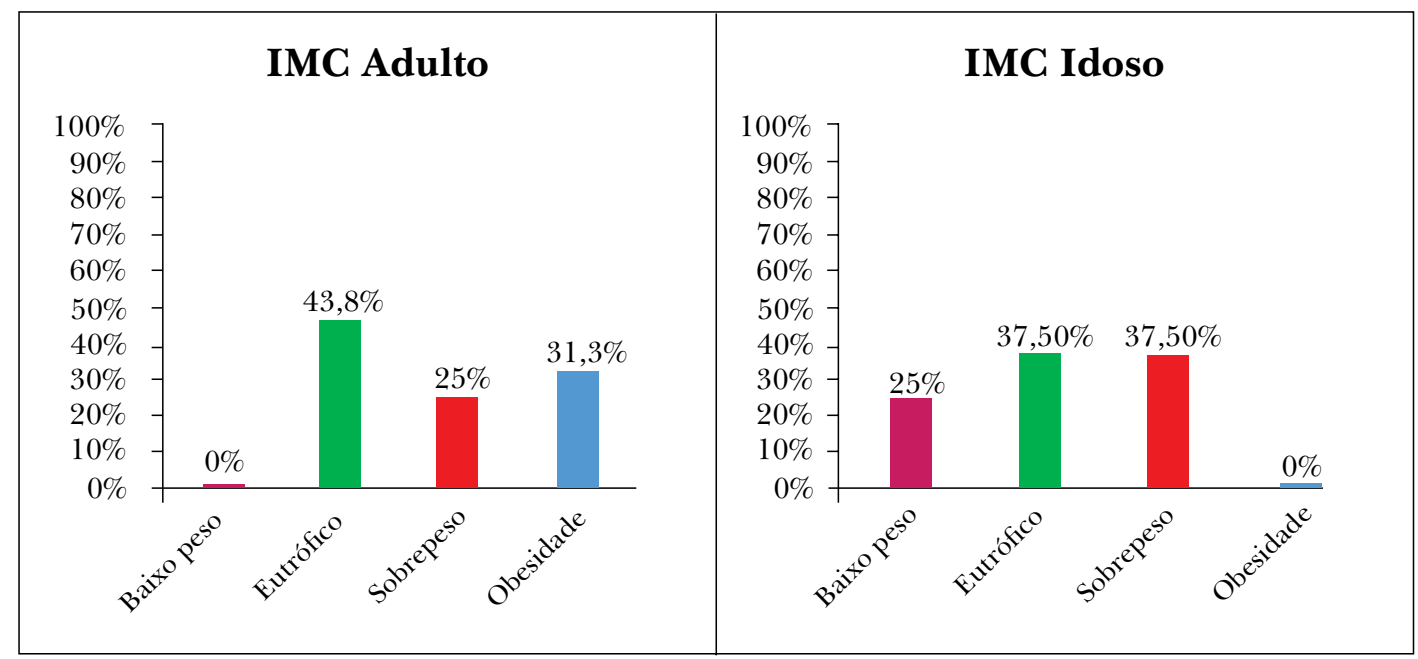

Figura 1. Estado nutricional antropométrico utilizando o IMC de pacientes adultos e idosos hospitalizados em Natal-RN, 2017.

Fonte: Dados da pesquisa. 
O estado nutricional segundo o IMC encontra-se na figura 1. Este não tem a capacidade de indicar a composição corporal, porém sua medida é realizada por ser de fácil mensuração e possuir relação com morbimortalidade.

No presente estudo, a prevalência de eutrofia se destaca na fase adulta, havendo declínio na fase da senescência. O sexo feminino apresentou maior IMC quando comparado ao público masculino, tendendo ao maior índice, conforme a idade. Barbosa et al., ${ }^{27} \mathrm{em}$ seu estudo, observaram redução do IMC conforme a idade e prevalência também no sexo feminino.

A mais alta prevalência de sobrepeso verificada entre as mulheres possivelmente se dá por mais acúmulo de gordura visceral. Durante o envelhecimento, há progressiva redistribuição da gordura, com diminuição do tecido adiposo subcutâneo dos membros e acúmulo na região intraabdominal. As mulheres acumulam mais gordura subcutânea que os homens e a perdem com o passar da idade. ${ }^{28}$ No geral, os idosos apresentaram equivalência de eutrofia e sobrepeso.

A composição corporal nessa idade está mais susceptível ao aumento da porcentagem de gordura corporal e redução da massa magra, mudanças antropométricas que geralmente são associadas à perda da força e da resistência muscular, podendo levar a um declínio das capacidades funcionais. ${ }^{29}$ Como mencionado, o IMC não diferencia a composição corporal; sendo assim, muitos dos pacientes hospitalizados apresentam classificação dentro da normalidade ou sobrepeso, e mesmo assim podem estar retratando um quadro de desnutrição ou edema associado.

Com relação aos dados do perímetro da panturrilha, a maioria dos adultos e idosos apresentouse como eutrófica em ambos os sexos, porém uma parte do público idoso encontrava-se em desnutrição. Rosa et al., ${ }^{30}$ em seu estudo, verificaram prevalência de desnutrição nos idosos internados em um hospital. Estes apresentavam, em sua maioria, risco de desnutrição, levando a crer que houve perda de massa muscular advinda de um processo catabólico e/ou decréscimo de atividade, já que o PP é um instrumento que fornece a medida mais sensível sobre a massa muscular do idoso, comparada à área muscular do braço. Podem-se observar os resultados oriundos do perímetro da panturrilha na figura 2. 


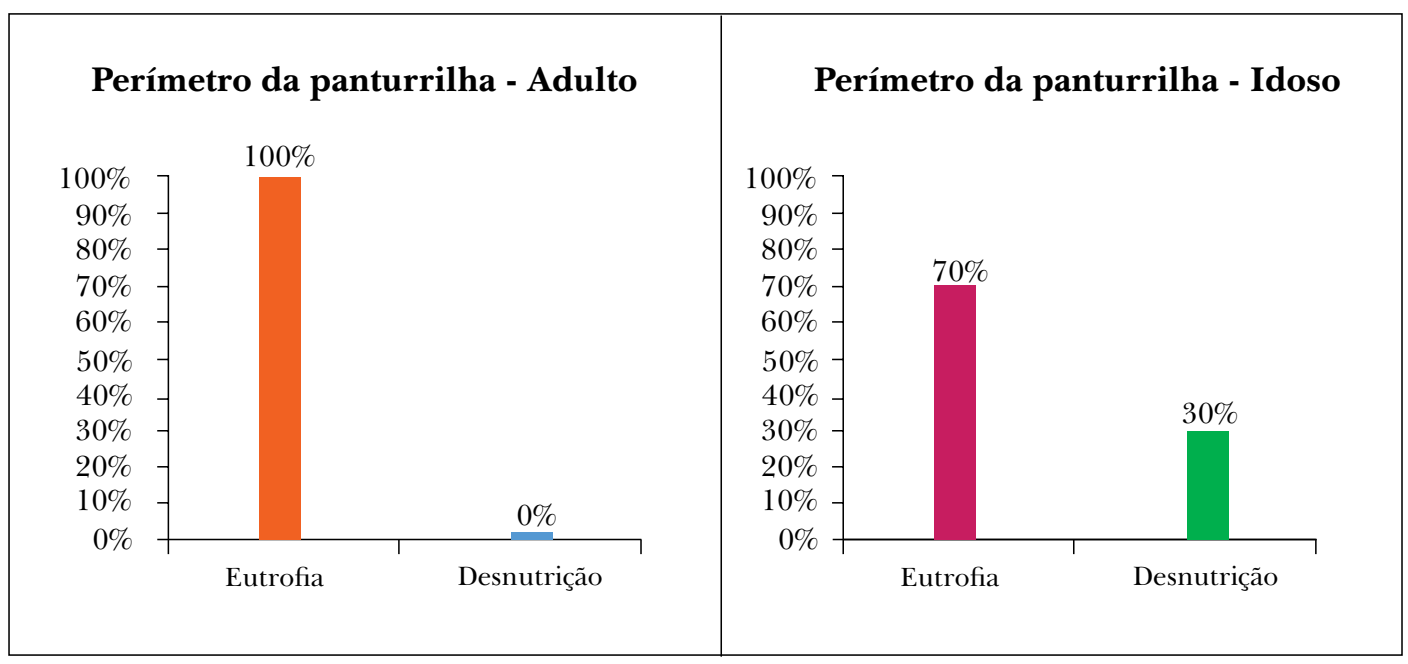

Figura 2. Estado nutricional antropométrico utilizando o perímetro da panturrilha de pacientes adultos e idosos hospitalizados em Natal-RN, 2017.

Fonte: Dados da pesquisa.

Ainda no estudo, verificou-se prevalência de eutrofia tanto no público adulto quanto idoso em relação ao PB, mas uma parcela apresentou desnutrição, variando de leve, moderado a grave. Ordoñez et al. ${ }^{31}$ observaram que, em idosos hospitalizados, dos parâmetros de avaliação do estado nutricional (IMC e PB), apenas a PB mostrou associação com o aumento da mortalidade. Além disso, resultados semelhantes foram descritos por Dent et al., ${ }^{32}$ que observaram associação de menor circunferência do PB com maior nível de assistência para idosos hospitalizados.

Alterações estruturais e funcionais, principalmente nos idosos, podem ocorrer, entre as quais a perda progressiva da massa muscular e modificações no padrão de distribuição da gordura corporal, no qual o tecido adiposo dos braços e das pernas diminui, aumentando o acúmulo de gordura na região do tronco, como já enfatizado. ${ }^{33}$ Os resultados obtidos são demonstrados na figura 3 . 


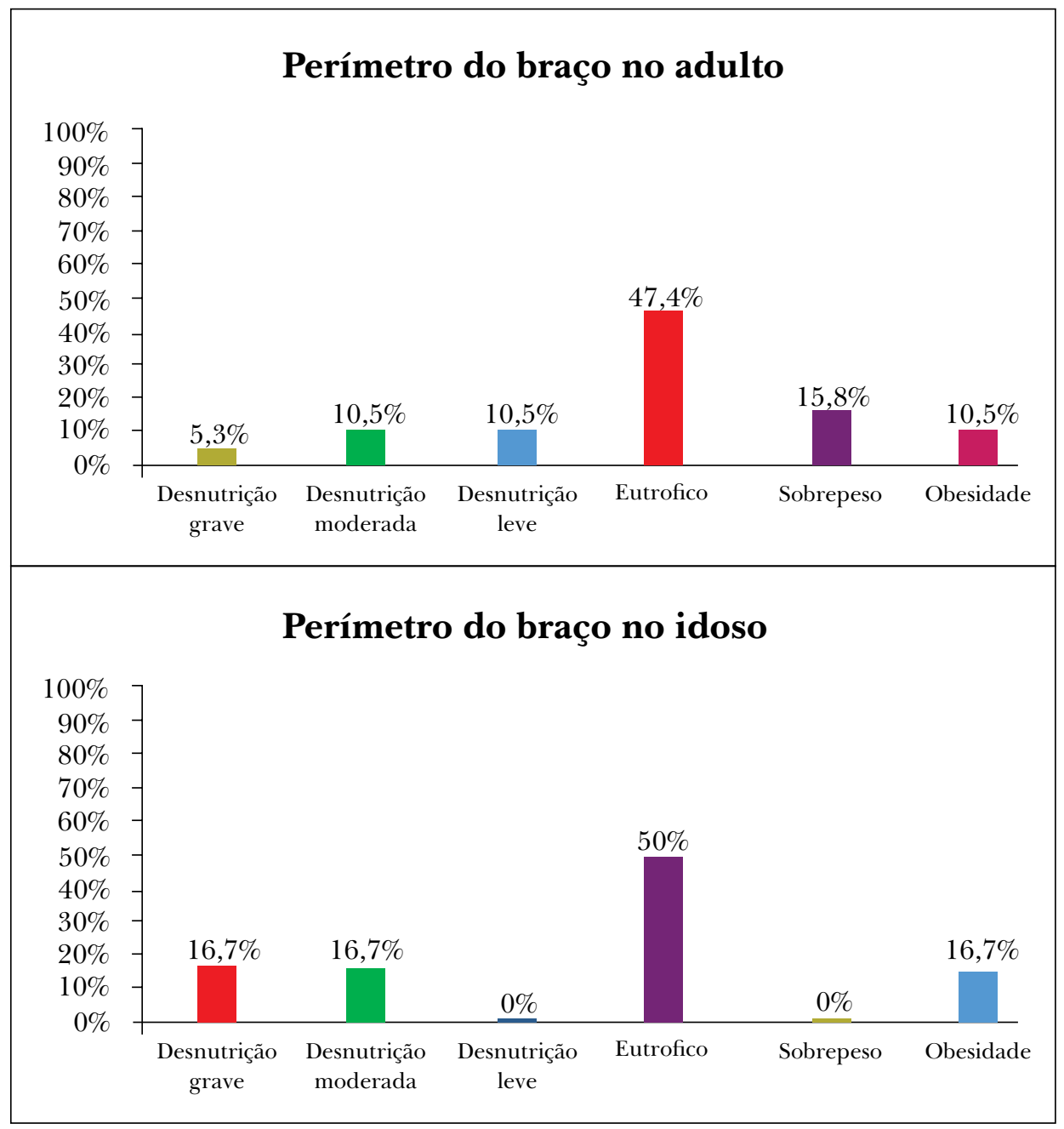

Figura 3. Estado nutricional antropométrico utilizando o perímetro do braço de pacientes adultos e idosos hospitalizados em Natal-RN, 2017.

Fonte: Dados da pesquisa. 
O EMAP apresentou maior índice de depleção nos adultos, quando comparados aos idosos, que no entanto apresentaram maior índice de depleção moderada e grave. Côbero et al. ${ }^{34}$ observaram fraca correlação do EMAP com o PB e IMC, apesar de a maioria encontrar-se com excesso de peso, segundo o IMC. Das amostras observadas, $50 \%$ apresentaram algum percentual de perda de peso e 22,3\% alteraram o consumo alimentar para dieta hipocalórica devido à diminuição do apetite. Isso demonstrou que, apesar da classificação nutricional encontrada, deve-se sempre atentar para o risco de desnutrição de pacientes hospitalizados.

As doenças crônicas, muitas vezes presentes nesses pacientes, resultam em diminuição das atividades e aumento do estado inflamatório e catabólico, ${ }^{35}$ associando-se, assim, à redução da troficidade muscular e, consequentemente, ao valor da EMAP.

Ainda no estudo citado, identificou-se que parte importante da amostra apresentava capacidade funcional física abaixo do normal. Provavelmente, a inatividade física agrava a redução da EMAP, independentemente do catabolismo e da doença básica do paciente. Pereira et al. ${ }^{9}$ afirmam que não foram observadas associações da EMAP com os parâmetros convencionais citados, pelo fato de o público ser restrito a pacientes em tratamento dialítico, que muitas vezes se apresentam edemaciados.

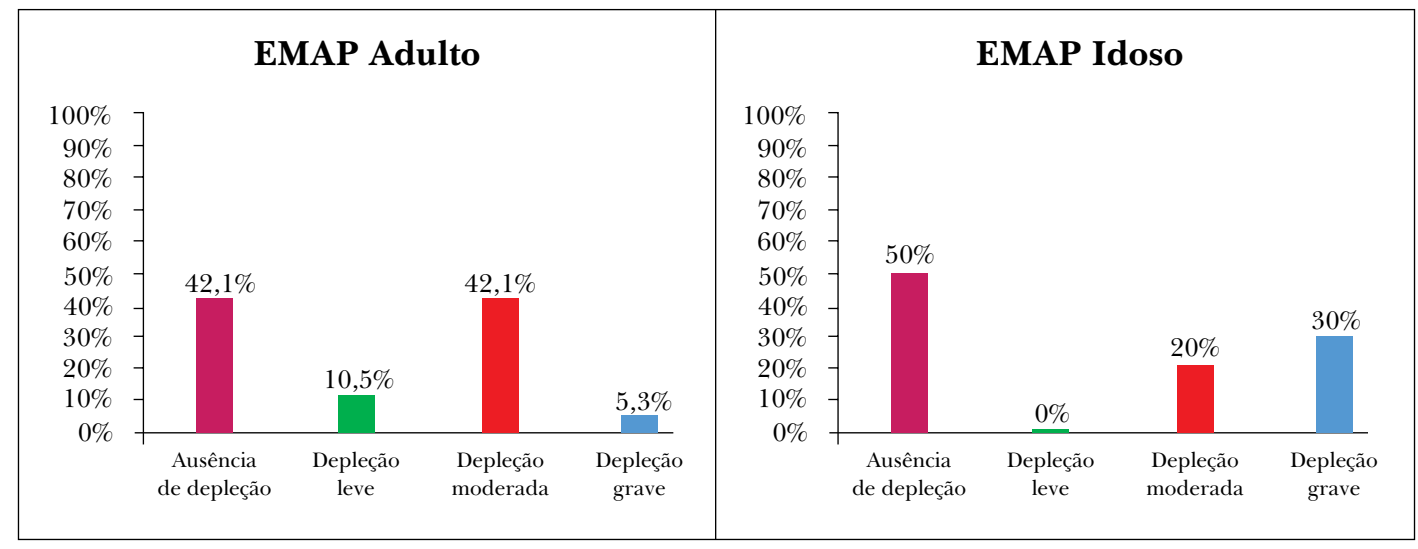

Figura 4. Estado nutricional antropométrico utilizando a espessura do músculo do adutor do polegar de pacientes adultos e idosos hospitalizados em Natal-RN, 2017.

Fonte: Dados da pesquisa. 
De acordo com a figura 5, verificou-se que a FPM para adultos apresentou elevado percentual de adequação nutricional, enquanto que no grupo de idosos o maior percentual foi de risco de depleção. As mulheres apresentaram FPM inferior e redução da força conforme a idade, assim como visto por diferentes estudos. ${ }^{27,36}$ Os homens apresentam maior massa muscular comparados às mulheres, e ambos apresentam redução desta conforme o avançar da idade (relação linear entre o processo de sarcopenia e a idade), ${ }^{37}$ justificando assim a redução do FPM no sexo feminino e maior risco de depleção nos idosos.

Lédo et al., ${ }^{38} \mathrm{em}$ seu trabalho analisando a sarcopenia em uma amostra de indivíduos infectados pelo vírus da imunodeficiência humana (HIV), verificaram que a FPP equivaleu a 21,3 \pm 6,4, quando comparados aos pacientes sem sarcopenia $(34,6 \pm 10,5)$. A perda de massa muscular esquelética associada à redução de força e/ou desempenho físico caracteriza essa condição. Esta se faz presente em muitos pacientes com risco clínico, sendo vista rotineiramente em idosos. Através da FPP, podemos vislumbrar a progressão das complicações das doenças, auxiliando principalmente quando associada a outros métodos para o diagnóstico da sarcopenia.

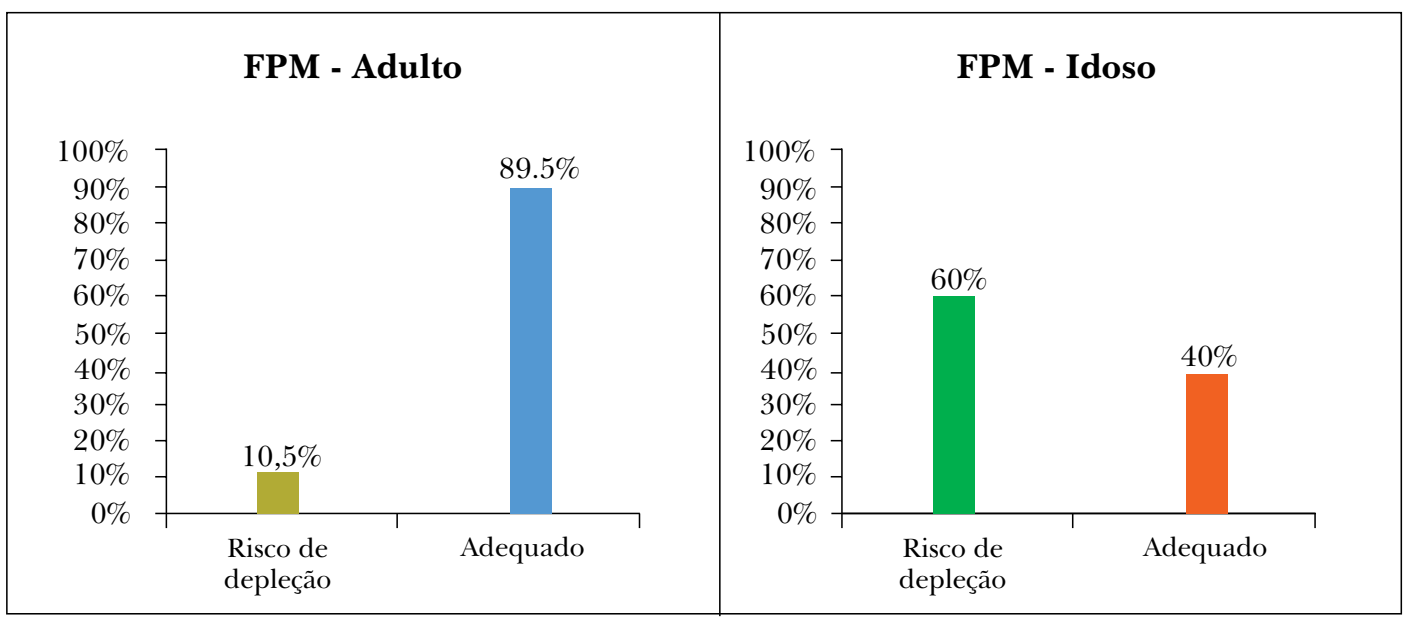

Figura 5. Estado nutricional antropométrico utilizando a força de preensão manual de pacientes adultos e idosos hospitalizados em Natal-RN, 2017.

Fonte: Dados da pesquisa.

\section{Conclusão}

Foi verificado que os pacientes internados no hospital público no período de coleta de dados encontraram-se eutróficos, quando avaliados tanto por métodos convencionais quanto não convencionais. Excetuaram-se a FPM nos indivíduos idosos e a MAP em ambas as populações que apresentaram risco para depleções. 
Sendo assim, os métodos não convencionais, principalmente quando associados aos convencionais, contribuíram para melhor visualização do estado nutricional dos pacientes hospitalizados.

Devido à escassez de estudos e à falta de parâmetros de classificação do estado nutricional de pacientes hospitalizados avaliados por métodos não convencionais, sugere-se realizar mais pesquisas que possam padronizar a classificação do estado nutricional, além de reconhecer pontos positivos e negativos nesses métodos não convencionais na avaliação nutricional em pacientes hospitalizados.

\section{Colaboradores}

Serquiz AC e Fernandes LL participaram da idealização do projeto, delineamento do estudo, orientação e acompanhamento nas coletas e revisão do artigo e versão final. Oliveira CM, Araújo ASM, Santos RD e Sousa JCS participaram do delineamento do estudo, coleta de dados, interpretação dos dados e redação do artigo.

Conflito de Interesses: Os autores declaram não haver conflito de interesses.

\section{Referências}

1. Bosco VEF, Sousa KMM, Viana KDAL, Calado IL. Estado nutricional de indivíduos hospitalizados em um hospital universitário de São Luís, Maranhão. Rev Pesq Saúde 2013; 14(1):21-26.

2. Fidelix MSP, Santana AFF, Gomes JR. Prevalência de desnutrição hospitalar em idosos. Revista da Associação Brasileira de Nutrição-RASBRAN 2013; 5(1):60-68.

3. Andrade PVB, Lameu EB. Espessura do músculo adutor do polegar: um novo índice prognóstico em pacientes clínicos. Rev Bras Nutr Clin. 2007; 22(1):28-35.

4. Saize MC, Simone CC, Filipe GS. Aplicação de métodos subjetivos e antropométricos na avaliação nutricional de adultos hospitalizados-uma revisão da literatura. Revista Rede de Cuidados em Saúde [Internet] 2013; 7(1). Disponível em: http://publicacoes.unigranrio.br/index.php/rcs/article/ view/1465/912

5. Waitzberg DL, Terra RM. Função muscular e sua relação com nutrição e desnutrição. In: Waitzberg DL. Nutrição oral, enteral e parenteral na prática clínica. 3. ed. São Paulo: Atheneu; 2006. p. 321-325.

6. Sande LP, Coury HJCG, Oishi J, Kumar S. Effect of muscoloskeletal disorders on prehension strength. Appl Ergon. 2001; 32(6):609-616.

7. Meldrum D, Cahalane E, Conroy R, Guthrie R, Hardiman O. Quantitative assessment of motor fatigue: normative values and comparison with prior-polio patients. Amyotroph Lateral Scler. 2007; 8(3):170-176.

8. Ikemoto Y, Demura S, Yamaji S, Minami M, Nakada M, Uchiyama M. Force-time parameters during explosive isometric grip correlate with muscle power. Sport Sci Health 2007; 2(2):64-70. 
9. Pereira RA, Caetano AL, Cuppari L, Kamimura MA. Espessura do músculo adutor do polegar como preditor da força de preensão manual nos pacientes em hemodiálise. J Bras Nefrol. 2013; 35(3):177-184.

10. Valente KP, Silva NMF, Barcelos AF, Barreto MA, Moraes RAG, Guandalini VR. Espessura do músculo adutor do polegar na avaliação nutricional de pacientes cirúrgicos. Einstein 2013; 14(1):18-24.

11. World Health Organization. Active ageing: a police framework. Geneva: WHO; 2002. 59 p.

12. Oliveira CMC, Kubrusly M, Mota RS, Choukroun G, Neto JB, Silva CAB. Adductor pollicis muscle thickness: a promising anthropometric parameter for patients witch chronic renal failure. J Ren Nutr. 2012; 22(3):307-316.

13. Webb AR, Newman LA, Taylor M, Keogh JB. Hand grip dynamometry as a predictor of postoperative complicationds reappraisal using age standardized grip strengths. J Parenter Enteral Nutr. 1989; 13(1):30-33.

14. Guigoz Y, Vellas B, Garry P. The mini nutrition assessment; a practical assessment tool for grading the nutritional state of elderly patients. In: Guigoz SC, et al. The Mini Nutritional Assessment: MNA, Facts and Research in Gerontology. New York: Serdi; 1999. p. 15-59.

15. Mussoi TD. Avaliação nutricional na prática clínica: da gestação ao envelhecimento. In: Mussoi TD. Avaliação do estado nutricional. Rio de Janeiro: Guanabara Koogan; 2014. p. 83.

16. Reis KS, Santana HS, Soares FB, Medeiros RS, Serrano HMS, Moreira SA. Prevalência de desnutrição em pacientes internados em um hospital geral. Revista Digital de Nutrição 2009; 3(5):477-488.

17. Lipschitz DA. Screening for nutritional status in the elderly. Prim Care 1994; 21:55-67.

18. Chumlea WC, Guo S, Roche AF, Steinbaugh ML. Prediction of body weight for the nonambulatory elderly from anthropometry. J Am Diet Assoc. 1988; 88(5):568-564.

19. Frisancho AR. Anthropometric standards for the assessment of growth and nutritional status. Ann Arbor: University of Michigan 1990.189 p.

20. Bragagnolo R, Caporossi FS, Dock-Nascimento DB, Aguilar-Nascimento JE, TCBC MT. Espessura do músculo adutor do polegar: um método rápido e confiável na avaliação nutricional de pacientes cirúrgicos. Rev Col Bras Cir Mato Grosso 2009; 5(36):371-376.

21. Mussoi TD. Avaliação nutricional na prática clínica: da gestação ao envelhecimento. In: Mussoi TD. Avaliação do estado nutricional. Rio de Janeiro: Guanabara Koogan; 2014. p. 134.

22. Soares ALG, Mussoi TO. Mini avaliação nutricional na determinação do risco nutricional e de desnutrição em idosos hospitalizados. Rev Bras Nutr Clin. 2014; 29(2):105-110.

23. Costa KMSM, Teixeira PS, Ferreira CB, Arruda AL, Mazzoccante RP, Ferreira AP. Perfil antropométrico funcional e cognitivo de idosos não institucionalizados. Rev Brasileira de Pesquisa em Ciências da Saúde 2017; 3(2):28-35.

24. Nascimento NC, Sostisso CF, Schieferdecker MEM, Rabito EI, Vilela RM. Comparação de métodos de detecção da desnutrição no ambiente hospitalar. Nutr Clín Diet Hosp. 2017; 37(1):34-40.

25. Matsudo SM, Matsudo VKR, Barros Neto TL. Impacto do envelhecimento nas variáveis antropométricas, neuromotoras e metabólicas da aptidão física. Revista Brasileira de Ciência e Movimento 2000; 8(4):21-32. 
26. Sperotto FM, Spinelli RB. Avaliação nutricional em idosos independentes de uma instituição de longa permanência no município de Erechim-RS. Perspectiva 2010; 34(125):105-116.

27. Barbosa AR, Souza JM, Lebrão ML, Marucci MDFN. Relação entre estado nutricional e força de preensão manual em idosos do município de São Paulo, Brasil: dados da pesquisa SABE. Rev Bras Cineantropom Desempenho Hum. 2006; 8(1):37-44.

28. World Health Organization. Physical status: the use and interpretation of anthropometry. Geneva: WHO; 1995. Technical report series, 854.

29. Rondanelli M, Talluri J, Peroni G, Donelli C, Guerriero F, Ferrini K, et al. Beyond body mass index. Is the Body Cell Mass Index (BCMI) a useful prognostic factor to describe nutritional, inflammation and muscle mass status in hospitalized elderly?: Body cell mass Index links in elderly. Clin Nutr. 2017; S0261-5614(17):30112-30117.

30. Rosa CB, Garces SBB, Hansen D, Brunelli AV, Bianchi PDA, Coser J, et al. Malnutrition risk and hospitalization in elderly assisted in Primary Care. Ciênc. Saúde Coletiva 2017; 22(2):575-582.

31. Ordoñez AM, Schieferdecker MEM, Cestonaro T, Cardoso Neto J, Campos ACL. Nutritional status influences the length of stay and clinical outcomes in patients hospitalized in internal medicine wards. Nutr Hosp. 2013; 28(4):1313-1320.

32. Dent E, Chapman I, Piantadosi C, Visvanathan R. Nutritional screening tools and anthropometric measures associate with hospital discharge outcomes in older people. Australas J Ageing 2015; 34(1):E1-6.

33. Menezes TN, Marucci MF. N. Antropometria de idosos residentes em instituições geriátricas, Fortaleza, CE. Rev Saúde Pública 2005; 39(2):169-175.

34. Cobêro FE, Gomes MCB, Silva AP, Bernardi JLD, McLellan KCP. A medida do músculo adutor do polegar está associada com indicadores antropométricos de avaliação de massa magra e de massa gorda em pacientes hospitalizados. Nutrire: Rev Soc Bras Alim Nutr. 2012; 37(2):174-182.

35. Guccione AA, Felson DT, Anderson JJ, Anthony JM, Zhang Y, Wilson PW, et al. The effects of specific medical conditions on the functional limitations of elders in the Framingham study. Am J Public Health 1994; 84(3):351-358.

36. Madsen OR, Lauridsen UR, Hartkopp A, Sorensen OH. Muscle strength and soft tissue composition as measured by dual-energy x-ray absorptiometry in women aged 18-87 Years. Eur J Appl Physiol Occup Physiol. 1997; 75(3):239-245.

37. Kuczmarski MF, Kuczmarski RJ, Najjar M. Descriptive anthropometric reference data for older Americans. J Am Diet Assoc. 2000; 100(1):59-66.

38. Lédo AP, Sá Neves J, Martinez BP, Gomes Neto M, Brites C. Sarcopenia em uma amostra de indivíduos infectados HIV atendidos a nível ambulatorial. Revista Pesquisa em Fisioterapia 2017; 7(3):400-407.

Recebido: 08 de junho, 2017

Revisado: 24 de outubro, 2017

Aceito: 21 de novembro, 2017 